\title{
ZKSCAN3 expression in urothelial carcinoma of the upper urinary tract and its impact on patient outcomes
}

\author{
Mehrsa Jalalizadeh ${ }^{1}$, Satoshi Inoue ${ }^{1-4}$, Kazutoshi Fujita ${ }^{1,5}$, Hiroki Ide ${ }^{1,4}$, Taichi Mizushima ${ }^{1-4}$, Seiji Yamaguchi ${ }^{6}$, Hiroaki Fushimi ${ }^{7}$, Norio
} Nonomura $^{5}$ and Hiroshi Miyamoto ${ }^{1-4,8^{*}}$

1James Buchanan Brady Urological Institute, The Johns Hopkins Hospital, USA

${ }^{2}$ Department of Pathology \& Laboratory Medicine, University of Rochester Medical Center, USA

3James P. Wilmot Cancer Center, University of Rochester Medical Center, USA

${ }^{4}$ Department of Pathology, Johns Hopkins University School of Medicine, USA

${ }^{5}$ Department of Urology, Osaka University Graduate School of Medicine, Japan

${ }^{6}$ Department of Urology, Osaka General Medical Center, Japan

${ }^{7}$ Department of Pathology, Osaka General Medical Center, Japan

${ }^{8}$ Departments of Urology, University of Rochester Medical Center, USA

\begin{abstract}
We recently found that a zinc-finger transcription factor, ZKSCAN3, could induce the growth of bladder cancer cells. In this study, we immunohistochemically stained for ZKSCAN3 in upper urinary tract urothelial carcinoma (UUTUC) specimens. ZKSCAN3 was positive in 52 [42.4\%; 26 (26.3\%) weak (1+), 13 (13.1\%) moderate $(2+)$, and $3(3.0 \%)$ strong $(3+)$ ] of 99 UUTUCs, which was significantly $(P<0.001)$ lower than in paired normal-appearing urothelial tissues [70 $(86.4 \%)$ of 81; 17 (21.0\%) weak, 36 (44.4\%) moderate, and 17 (21.0\%) strong]. There were no statistically significant associations between ZKSCAN3 expression pattern and tumor grade, $\mathrm{pT} / \mathrm{pN}$ stage, or distant metastasis. However, the positive rate of $Z$ KSCAN3 expression was significantly $(P<0.001)$ higher in ureteral tumors $(54.0 \%)$ than in renal pelvic tumors (26.7\%). Kaplan-Meier and log-rank tests revealed that the levels of ZKSCAN expression did not considerably correlate with progressionfree or cancer-specific survival in the entire cohort of the patients. Meanwhile, strong (3+) ZKSCAN3 expression in muscle-invasive tumor was correlated with the risk of disease progression $(P=0.001)$ and cancer-specific mortality $(P=0.069)$, while only one patient had a $Z$ KSCAN3(3+) muscle-invasive tumor. Thus, in contrast to the observations in bladder specimens, the decreased expression of ZKSCAN3 in UUTUC, compared with non-neoplastic urothelium, was seen. Moreover, the current results failed to provide conclusive evidence suggesting the prognostic value of ZKSCAN3 expression in patients with UUTUC.
\end{abstract}

\section{Introduction}

Upper urinary tract urothelial carcinoma (UUTUC) accounts for less than $10 \%$ of urothelial cancers but is often aggressive with poor prognosis compared with bladder cancer [1]. Presumably due to the scarcity of UUTUC, the pathophysiology of this disease is not well understood, while some of molecular or genetic factors are similar to those associated with bladder cancer [1,2]. Importantly, further validation is still required to determine the prognostic values of current molecular markers for UUTUC, other than histopathological features including tumor grade and stage, lymphovascular invasion, surgical margin status, and the presence of concomitant carcinoma in situ [2].

ZKSCAN3, a family member of the KRAB and SCAN domaincontaining zinc-finger transcription factors, has been shown to involve the outgrowth of malignancies [3-6]. It has indeed been documented that overexpression or gene amplification of ZKSCAN3 in tissue specimens correlates with colorectal cancer invasion [4] or prostate cancer metastasis [4], respectively. Recently, we demonstrated that ZKSCAN3 knockdown in bladder cancer lines resulted in considerable decreases in cell viability, along with increased apoptosis, as well as in cell migration and invasion [6]. Additionally, in xenograft-bearing mice, ZKSCAN3 knockdown significantly delayed tumor formation and subsequent tumor growth [6]. Thus, ZKSCAN3 activation appeared to contribute to the promotion of tumor progression in several types of malignancies, including bladder cancer. However, little is known about the functional role of ZKSCAN3 signaling in the development and progression of UUTUC. The current study aims to determine the expression status of ZKSCAN3 in UUTUCs and corresponding nonneoplastic urothelial tissues as well as to assess prognostic significance of ZKSCAN3 expression.

\section{Materials and methods}

UUTUC tissue microarray (TMA) was constructed with tumor samples and paired normal-appearing urothelial tissues from 99 patients who underwent radical nephroureterectomy performed at Osaka General Medical Center, Osaka, Japan, as described previously $[7,8]$. Appropriate approval was obtained from the institutional review board before construction and use of the TMA.

Correspondence to: Hiroshi Miyamoto, MD, $\mathrm{PhD}$, Department of Pathology \& Laboratory Medicine, University of Rochester Medical Center, 601 Elmwood Avenue, Box 626, Rochester, NY 14642, USA, Tel: +1(585)275-8748, E-mail: hiroshi_miyamoto@urmc.rochester.edu

key words: ZKSCAN3, immunohistochemistry, prognosis, upper urinary tract urothelial carcinoma

Received: May 19, 2017; Accepted: June 16, 2017; Published: June 19, 2017 
Immunohistochemical staining was performed on the sections ( $5 \mu \mathrm{m}$ thick) from the UUTUC TMA, using a primary antibody to ZKSCAN3 (TA308508; dilution 1:50; OriGene), as we described previously $[6,8]$. All the stains were manually scored by a single pathologist (H.M.) who was blinded to sample identity. The German Immunoreactive Score (range: 0 -12) calculated by multiplying the percentage of immunoreactive cells $(0 \%=0 ; 1-10 \%=1 ; 11-50 \%=2$; $51-80 \%=3 ; 81-100 \%=4)$ by staining intensity $(0$, negative; 1 , weak; 2 , moderate; 3 , strong) was considered negative $(0 ; 0-1)$, weakly positive $(1+; 2-4)$, moderately positive (2+;6-8), or strongly positive (3+;9-12).

The Fisher's exact test was used to evaluate the association between categorized variables. The survival rates were determined using the Kaplan-Meier method and comparison was made by the log-rank test. Tumor progression was defined as the development of nonbladder lesions, including recurrence at the nephroureterectomy site and lymph node or visceral metastasis. $P$ values less than 0.05 were considered statistically significant.

\section{Results}

We immunohistochemically stained for ZKSCAN3 in 99 UUTUC samples and corresponding 81 normal-appearing urothelial tissues. Positive signals for ZKSCAN3 were detected predominantly in the nucleus of benign and malignant epithelial cells (Figure 1).

Table 1 summarizes the status of ZKSCAN3 expression in nonneoplastic urothelium versus urothelial tumor tissues. ZKSCAN3 was positive in $70(86.4 \%)$ of 81 benign urothelial tissue samples [17 (21.0\%) 1+, $36(44.4 \%) 2+$, and $17(21.0 \%) 3+]$ and $42(42.4 \%)$ of 99 UUTUCs [26 (26.3\%) 1+, $13(13.1 \%) 2+$, and $3(3.0 \%) 3+$ ]. Thus, the levels of ZKSCAN3 expression were significantly reduced in tumor compared with non-neoplastic urothelium $(P<0.001)$.

Next we analyzed the correlations between the status of ZKSCAN3 expression in UUTUCs and the clinicopathological profile available for our patient cohort (Table 2). There were no strong associations between ZKSCAN3 expression pattern and the laterality of tumors, gender of the patients (data not shown), histopathological tumor grade, $\mathrm{pT}$ stage, $\mathrm{pN}$ stage, or distant metastasis (data not shown). However, the positive rate of ZKSCAN3 expression was significantly higher $(P=0.012)$ in ureteral tumors compared with renal pelvic tumors.

We then performed Kaplan-Meier analysis coupled with the logrank test to assess the prognostic values of ZKSCAN3 expression in UUTUCs. There were no statistically significant differences in tumor progression ( 0 vs. $1+/ 2+/ 3+, P=0.361$, Figure $2 \mathrm{~A} ; 0 / 1+$ vs. $2+/ 3+$, $P=0.696 ; 0 / 1+/ 2+v s .3+, P=0.969)$, tumor recurrence in the bladder (0 vs. $1+/ 2+/ 3+, P=0.802 ; 0 / 1+$ vs. $2+/ 3+, P=0.565 ; 0 / 1+/ 2+$ vs. $3+$, $P=0.346)$, or cancer-specific mortality $(0$ vs. $1+/ 2+/ 3+, P=0.406$, Figure $2 \mathrm{~B} ; 0 / 1+v s .2+/ 3+, P=0.879 ; 0 / 1+/ 2+v s .3+, P=0.772)$ between dichotomized levels of ZKSCAN3 expression in the entire cohort of the patients. Similarly, in 62 patients with muscle-invasive UUTUC, ZKSCAN3 positivity ( 0 vs. $1+/ 2+/ 3+)$ was not significantly associated with tumor progression $(P=0.122$, Figure $2 \mathrm{C})$ or cancer-specific mortality $(P=0.307$, Figure $2 \mathrm{D})$. However, there was a link between strong (3+) ZKSCAN3 expression in a muscle-invasive UUTUC and the risk of tumor progression $(P=0.001$, Figure $2 \mathrm{E})$ or cancer-specific mortality $(P=0.069$; Figure $2 \mathrm{~F})$.

\section{Discussion}

In the present study, we immunohistochemically determined the expression levels of ZKSCAN3 in a set of TMA consisting of

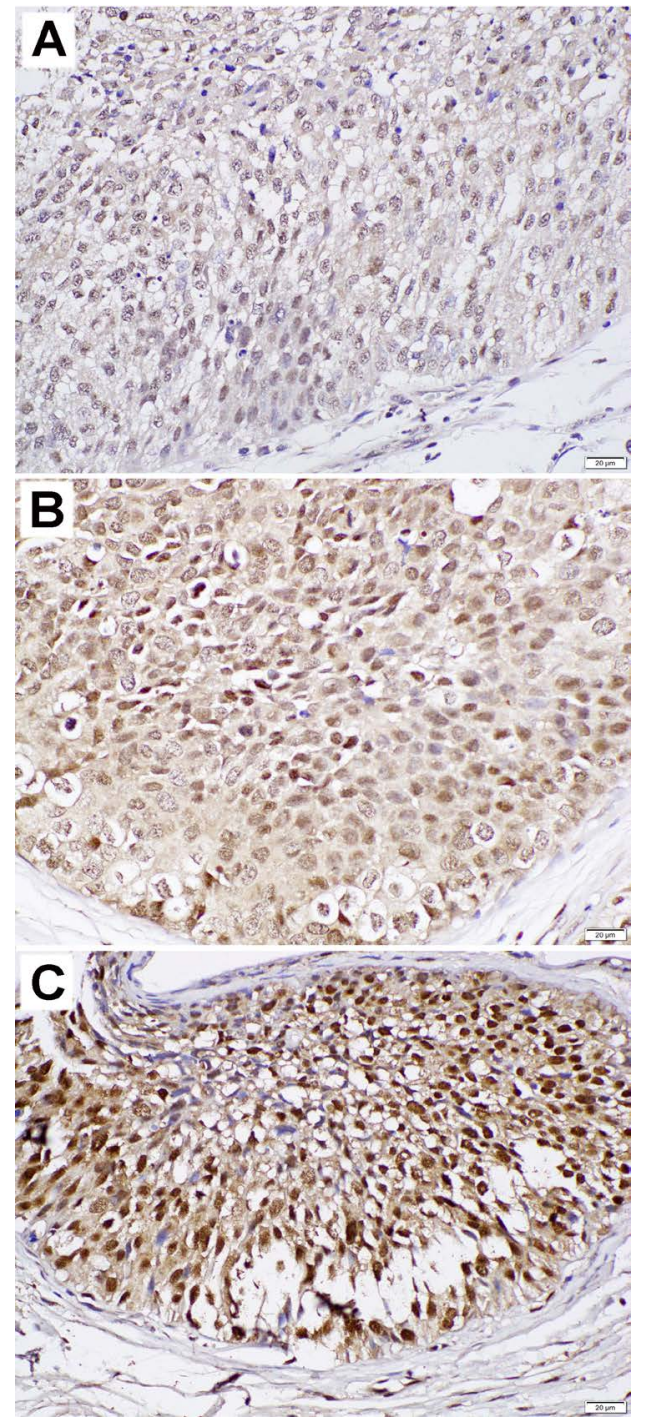

Figure 1. Immunohistochemistry of ZKSCAN3 in urothelial tumor tissue specimens (original magnification: $\times 400$ ). The staining is scored by a combination of the intensity $[$ i.e. weak (A), moderate (B), strong (C)] and distribution (i.e. percentage of immunoreactive cells).

nephroureterectomy specimens. We obtained some results similar to previous observations in bladder cancer tissue samples and others dissimilar to them.

We previously showed, in bladder specimens using the same staining/scoring methods, that ZKSCAN3 expression was elevated in bladder cancers [138 (93.2\%) of 148: 44 (29.7\%) weak, 55 (37.2\%) moderate, and 39 (26.4\%) strong], compared with non-neoplastic portions of urothelial tissues [76 (84.4\%) of 90: 27 (30.0\%) weak, 36 (40.0\%) moderate, and $13(14.4 \%)$ strong] [6]. In contrast to these previous findings, the levels of ZKSCAN3 expression was found to be considerably lower in UUTUCs than in non-neoplastic urothelial tissues. However, the status of ZKSCAN3 expression in benign urothelium from nephroureterectomy specimens appeared to be similar to that from bladder specimens in our previous study [6]. In addition, these normal-appearing tissues used for previous and current immunohistochemical stains were from surgical specimens from patients with bladder cancer and UUTUC, respectively, and therefore may not represent completely normal controls. Moreover, because 

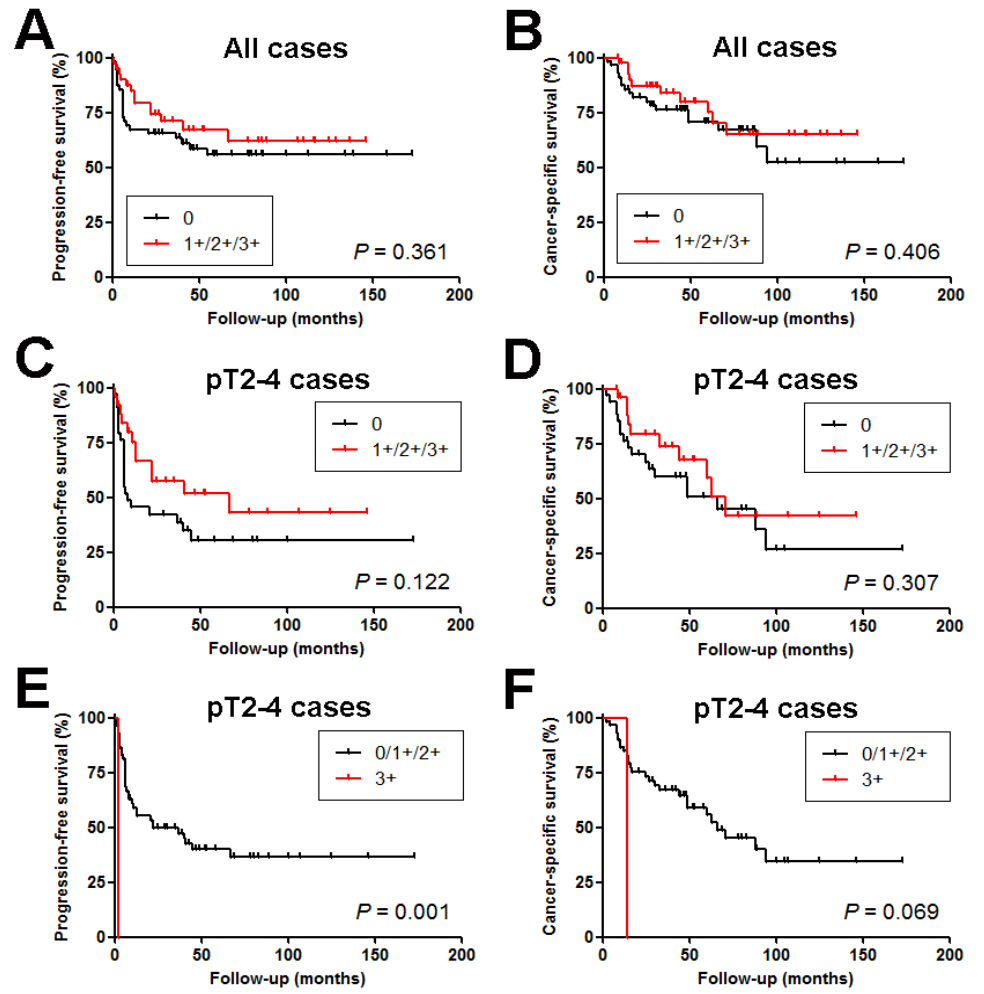

Figure 2. Progression-free survival (A, C, E) or cancer-specific survival (B, D, F) in 99 patients with UUTUC (A, B) or 62 patients with muscle-invasive UUTUC (C-F), according to the levels of ZKSCAN3 expression (A-D: 0 vs. 1+/2+3+; E, F: 0/1+/2+ vs. 3+).

Table 1. ZKSCAN3 expression in non-neoplastic urothelium and urothelial neoplasm tissue specimens

\begin{tabular}{|c|c|c|c|c|c|c|c|c|}
\hline \multirow{2}{*}{ Tissue } & \multirow{2}{*}{$\mathbf{n}$} & \multicolumn{4}{|c|}{ ZKSCAN3 expression } & \multicolumn{2}{|c|}{$\boldsymbol{P}$ value } \\
\cline { 2 - 7 } & & $0(\%)$ & $1+(\%)$ & $2+(\%)$ & $3+(\%)$ & $0 v s .1+/ 2+/ 3+$ & $0 / 1+v s .2+/ 3+$ & $0 / 1+/ 2+v s .3+$ \\
\hline Normal urothelium & 81 & $11(13.6)$ & $17(21.0)$ & $36(44.4)$ & $17(21.0)$ & $<0.001$ & $<0.001$ \\
\hline Urothelial neoplasm & 99 & $57(57.6)$ & $26(26.3)$ & $13(13.1)$ & $3(3.0)$ & $<0.001$ \\
\hline
\end{tabular}

Table 2. Correlations between ZKSCAN3 expression and clinicopathological profile of the patients

\begin{tabular}{|c|c|c|c|c|c|c|c|c|}
\hline \multirow{2}{*}{ Parameter } & \multirow{2}{*}{$\mathbf{n}$} & \multicolumn{4}{|c|}{ ZKSCAN3 expression } & \multicolumn{3}{|c|}{$P$ value } \\
\hline & & $0(\%)$ & $1+(\%)$ & $2+(\%)$ & $3+(\%)$ & 0 vs. $1+/ 2+/ 3+$ & $0 / 1+v s .2+/ 3+$ & $0 / 1+/ 2+v s .3+$ \\
\hline Laterality & & & & & & 0.153 & 0.106 & 0.578 \\
\hline Right & 43 & $21(48.8)$ & $12(27.9)$ & $8(18.6)$ & $2(4.7)$ & & & \\
\hline Left & 56 & $36(64.3)$ & $14(25.0)$ & $5(8.9)$ & $1(1.8)$ & & & \\
\hline Tumor site & & & & & & $0.012^{\mathrm{a}}$ & $0.180^{\mathrm{a}}$ & $0.244^{\mathrm{a}}$ \\
\hline Renal pelvis & 45 & $33(73.3)$ & $7(15.6)$ & $5(11.1)$ & $0(0)$ & & & \\
\hline Ureter & 50 & $23(46.0)$ & $16(32.0)$ & $8(16.0)$ & $3(6.0)$ & & & \\
\hline Both & 4 & $1(25.0)$ & $3(75.0)$ & $0(0)$ & $0(0)$ & & & \\
\hline Tumor grade & & & & & & 1.000 & 0.705 & 1.000 \\
\hline Low-grade carcinoma & 15 & $9(60.0)$ & $3(20.0)$ & $3(20.0)$ & $0(0)$ & & & \\
\hline High-grade carcinoma & 84 & $48(57.1)$ & $23(27.4)$ & $10(11.9)$ & $3(3.6)$ & & & \\
\hline Pathologic stage & & & & & & $0.835^{\mathrm{b}}$ & $1.000^{\mathrm{b}}$ & $0.554^{\mathrm{b}}$ \\
\hline pTa & 19 & $13(68.4)$ & $3(15.8)$ & $2(10.5)$ & $1(5.3)$ & & & \\
\hline pT1 & 18 & $9(50.0)$ & $6(33.3)$ & $2(11.1)$ & $1(5.6)$ & & & \\
\hline Non-muscle-invasive & 37 & $22(59.5)$ & $9(24.3)$ & $4(10.8)$ & $2(5.4)$ & & & \\
\hline pT2 & 8 & $4(50.0)$ & $3(37.5)$ & $1(12.5)$ & $0(0)$ & & & \\
\hline pT3 & 48 & $27(56.3)$ & $14(29.2)$ & $6(12.5)$ & $1(2.1)$ & & & \\
\hline pT4 & 6 & $4(66.7)$ & $0(0)$ & $2(33.3)$ & $0(0)$ & & & \\
\hline Muscle-invasive & 62 & $35(56.5)$ & $17(27.4)$ & $9(14.5)$ & $1(1.6)$ & & & \\
\hline Lymph node involvement & & & & & & $0.548^{\mathrm{c}}$ & $0.684^{c}$ & $1.000^{c}$ \\
\hline pNO & 84 & $47(56.0)$ & $23(27.4)$ & $11(13.1)$ & $3(3.6)$ & & & \\
\hline $\mathrm{pN} 1-3$ & 12 & $8(66.7)$ & $3(25.0)$ & $1(8.3)$ & $0(0)$ & & & \\
\hline $\mathrm{pNx}$ & 3 & $2(66.7)$ & $0(0)$ & $1(33.3)$ & $0(0)$ & & & \\
\hline
\end{tabular}

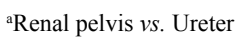

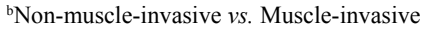

cpN0 vs. pN1-3 
there was a significant decrease in the positive rate of ZKSCAN3 expression in renal pelvic tumors (26.7\%), compared with ureteral tumors $(54.0 \%)$, differences in tissue preservation among bladder, ureteral, and renal pelvic tumors due to their anatomic locations and/ or thickness of the specimens around the tumors (e.g. time to complete tissue fixation) might have had an impact on the immunoreactivity. Of note, delay to formalin fixation of surgical specimens is known to lead to false-negative results in immunohistochemistry of transcription factors (e.g. estrogen receptor or progesterone receptor expression in breast cancer [9]). Thus, the positive rate of ZKSCAN3 expression in UUTUC may have been underestimated in the current study. Indeed, a similar tendency was seen in the expression of androgen receptor (i.e. $42 \%$ in bladder tumors [10] vs. $28 \%$ in ureteral tumors [8] vs. $11 \%$ in renal pelvic tumors [8]) as well as another transcription factor GATA3 (i.e. $86 \%$ in bladder tumors [11] vs. $68 \%$ in ureteral tumors [12] vs. $36 \%$ in renal pelvic tumors [12]) we stained using the same protocol with identical antibodies. Meanwhile, we found no significant correlations of ZKSCAN3 expression with tumor grade or stage in both UUTUC and bladder cancer.

No prognostic significance of ZKSCAN3 expression was identified in the stains of the bladder TMA [6]. Similarly, ZKSCAN3 positivity was not strongly associated with the prognosis of the entire cohort of patients with UUTUC or that in a subgroup of patients with muscleinvasive UUTUC. Nonetheless, a single case of muscle-invasive UUTUC showing strong (3+) ZKSCAN3 positivity was found to have higher risks of disease progression and cancer-specific mortality. Thus, we failed to provide conclusive evidence suggesting that ZKSCAN3 expression serves as a prognosticator in UUTUC patients, while ZKSCAN3 signals have been shown to promote the progression of urothelial tumors in preclinical models [6].

In conclusion, we observed a significant decrease in the expression levels of ZKSCAN3 in UUTUC, compared with non-neoplastic urothelium included in the same surgical specimens, as well as in those in renal pelvic tumors, compared with ureteral tumors. The clinicopathological and prognostic significance of ZKSCAN3 expression in urothelial cancer needs to be further determined in larger patient cohorts of UUTUC and bladder cancer as well as completely normal urothelial tissues. Further assessments of ZKSCAN3 functions are also required to determine its biological significance in the development and progression of UUTUC.

\section{References}

1. Raman JD, Messer J, Sielatycki JA, Hollenbeak CS (2011) Incidence and survival of patients with carcinoma of the ureter and renal pelvis in the USA, 1973-2005. BJU Int 107: 1059-1064. [Crossref]

2. Mbeutcha A, Rouprêt M, Kamat AM, Karakiewicz PI, Lawrentschuk N, et al. (2017) Prognostic factors and predictive tools for upper tract urothelial carcinoma: a systematic review. World J Urol 35: 337-353. [Crossref]

3. Yang L, Hamilton SR, Sood A, Kuwai T, Ellis L, et al. (2008) The previously undescribed ZKSCAN3 (ZNF306) is a novel "driver" of colorectal cancer progression. Cancer Res 68: 4321-4330. [Crossref]

4. Zhang X, Jing Y, Qin Y, Hunsucker S, Meng H, et al. (2012) The zinc finger transcription factor ZKSCAN3 promotes prostate cancer cell migration. Int $J$ Biochem Cell Biol 44: 1166-1173. [Crossref]

5. Lupo A, Cesaro E, Montano G, Zurlo D, Izzo P, et al. (2013) KRAB-Zinc Finger Proteins: A Repressor Family Displaying Multiple Biological Functions. Curr Genomics 14: 268-278. [Crossref]

6. Kawahara T, Inoue S, Ide H, Kashiwagi E, Ohtake S, et al. (2016) ZKSCAN3 promotes bladder cancer cell proliferation, migration, and invasion. Oncotarget 7: 53599-53610. [Crossref]

7. Munari E, Fujita K, Faraj S, Chaux A, Gonzalez-Roibon N, et al. (2013) Dysregulation of mammalian target of rapamycin pathway in upper tract urothelial carcinoma. Hum Pathol 44: 2668-2676. [Crossref]

8. Kashiwagi E, Fujita K, Yamaguchi S, Fushimi H, Ide H, et al. (2016) Expression of steroid hormone receptors and its prognostic significance in urothelial carcinoma of the upper urinary tract. Cancer Biol Ther 17: 1188-1196. [Crossref]

9. Neumeister VM, Anagnostou V, Siddiqui S, England AM, Zarrella ER, et al. (2012) Quantitative assessment of effect of preanalytic cold ischemic time on protein expression in breast cancer tissues. J Natl Cancer Inst 104: 1815-1824. [Crossref]

10. Miyamoto H, Yao JL, Chaux A, Zheng Y, Hsu I, et al. (2012) Expression of androgen and oestrogen receptors and its prognostic significance in urothelial neoplasm of the urinary bladder. BJU Int 109: 1716-1726. [Crossref]

11. Miyamoto H, Izumi K, Yao JL, Li Y, Yang Q, et al. (2012) GATA binding protein 3 is down-regulated in bladder cancer yet strong expression is an independent predictor of poor prognosis in invasive tumor. Hum Pathol 43: 2033-2040. [Crossref]

12. Inoue S, Mizushima T, Fujita K, Meliti A, Ide H, et al. (2017) GATA3 immunohistochemistry in urothelial carcinoma of the upper urinary tract as a urothelial marker as well as a prognosticator. Hum Pathol. [Crossref]

Copyright: (C2017 Jalalizadeh M. This is an open-access article distributed under the terms of the Creative Commons Attribution License, which permits unrestricted use, distribution, and reproduction in any medium, provided the original author and source are credited. 